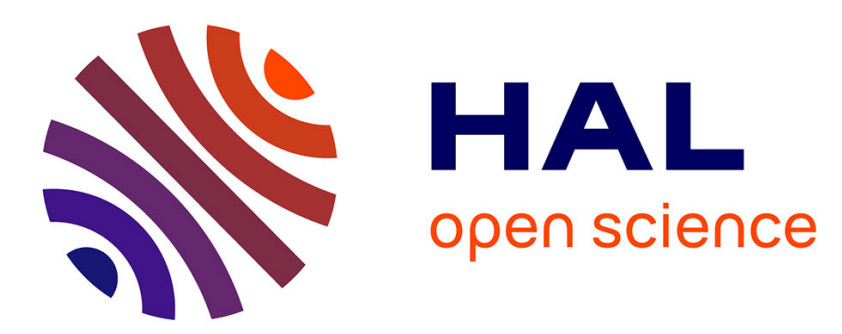

\title{
Numerical Workflow of Irreversible Electroporation for Deep-Seated Tumor
}

Olivier Gallinato, Baudouin Denis de Senneville, Olivier Seror, Clair Poignard

\section{To cite this version:}

Olivier Gallinato, Baudouin Denis de Senneville, Olivier Seror, Clair Poignard. Numerical Workflow of Irreversible Electroporation for Deep-Seated Tumor. Physics in Medicine and Biology, 2019, 64 (5), pp.055016. 10.1088/1361-6560/ab00c4 . hal-02063020

\section{HAL Id: hal-02063020 \\ https://hal.inria.fr/hal-02063020}

Submitted on 10 Mar 2019

HAL is a multi-disciplinary open access archive for the deposit and dissemination of scientific research documents, whether they are published or not. The documents may come from teaching and research institutions in France or abroad, or from public or private research centers.
L'archive ouverte pluridisciplinaire HAL, est destinée au dépôt et à la diffusion de documents scientifiques de niveau recherche, publiés ou non, émanant des établissements d'enseignement et de recherche français ou étrangers, des laboratoires publics ou privés. 


\title{
NUMERICAL WORKFLOW OF IRREVERSIBLE ELECTROPORATION FOR DEEP-SEATED TUMOR
}

\author{
OLIVIER GALLINATO*, BAUDOUIN DENIS DE SENNEVILLE* ${ }^{\star}$ OLIVIER SEROR ${ }^{\dagger}$, \\ AND CLAIR POIGNARD ${ }^{\star, \ddagger}$
}

\section{Contents}

$\begin{array}{ll}1 . & \text { Introduction }\end{array}$

1.1. IRE ablation for liver tumors 2

$\begin{array}{lll}1.2 . & \text { Numerical treatment planning for IRE } & 3\end{array}$

\begin{tabular}{lll}
\hline 1.3. & Goal of the paper & 3
\end{tabular}

2. Material and Methods 4

2.1. Clinical workflow and data collection 4

2.2. Case report 5

$2.3 . \quad$ Image Processing 5

\begin{tabular}{|ll}
$2.4 . \quad$ Numerical electroporation model & 6 \\
\hline
\end{tabular}

\begin{tabular}{lrr}
\hline 3. & Results & 8
\end{tabular}

\begin{tabular}{lll}
\hline 3.1. & Generic numerical workflow & 8
\end{tabular}

\begin{tabular}{llr}
\hline 3.2. & Patient-specific study & 9 \\
\hline
\end{tabular}

\begin{tabular}{ll}
\hline $4 . \quad$ Discussion & 12
\end{tabular}

4.1. Choice of the electroporation model and the numerical method 12

4.2. Numerical workflow: a tool for an accurate EF distribution 12

4.3. Issue of treatment assessment 13

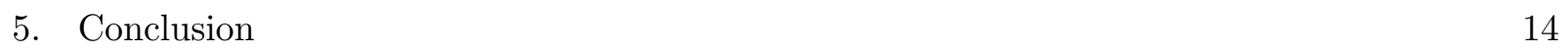

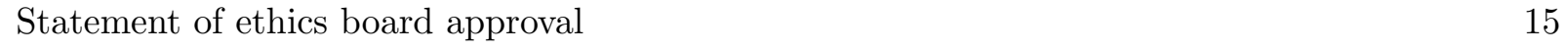

\begin{tabular}{|ll}
\hline Acknowledgement & 15
\end{tabular}

\begin{tabular}{ll}
\hline References & 15
\end{tabular}

$\left(^{\star}\right)$ INRIA Bordeaux-Sud-Ouest, CNRS, Bordeaux INP, Univ. Bordeaux, IMB, UMR 5251, F-33400, Talence, France

$\left(^{\dagger}\right)$ Department of Radiology, Hôpital Jean Verdier, Hôpitaux Universitaires Paris-Seine-SaintDenis, Assistance Publique Hôpitaux de Paris, Avenue du 14 julllet, 93140 Bondy, France UMR 1162, Génomique Fonctionnelle des Tumeurs solides, inserm, Paris, France

E-mail addresses: clair.poignard@inria.fr $\left({ }^{\ddagger}\right.$ :Corresponding author), olivier.gallinato@inria.fr. 
ABSTRACT. The paper provides a numerical workflow, based on the "real-life" clinical workflow of irreversible electroporation (IRE) performed for the treatment of deep-seated liver tumors. Thanks to a combination of numerical modeling, image registration algorithm and clinical data, our numerical workflow enables to provide the distribution of the electric field as effectively delivered by the clinical IRE procedure. As a proof of concept, we show on a specific clinical case of IRE ablation of liver tumor that clinical data could be advantageously combined to numerical simulations in a near future, in order to give to the interventional radiologists information on the effective IRE ablation. We also corroborate the simulated treated region with the post-treatment MRI performed 3 days after the treatment.

\section{INTRODUCTION}

Irreversible electroporation (IRE) is a minimally invasive and mainly nonthermal cancer therapy [6, 34, 36], which has emerged for the last decade among the promising non surgical local ablation techniques for solid tumors [47, 3, 38, 29, 41]. IRE ablation is a focal energy-based method, which consists in delivering high voltage short pulses to the tumor. More precisely several tens of high voltage pulses of one hundred microseconds are delivered between each needle pair surrounding the target tumor. The rationale of IRE states that if the electric field (EF) magnitude reaches about $600 \mathrm{~V} / \mathrm{cm}$ [6] for a train of micropulses, then cell membranes are irreversibly damaged by the electric field -leading to cell apoptosis- while the surrounding tissue scaffold is preserved [35]. Therefore IRE is seen as the treatment of choice for tumors located near vital structures (veins, arteries, bile ducts) [21, 19, 4]. Indeed, the safety and efficiency of IRE has been shown for patients with lesions smaller than $5 \mathrm{~cm}$ and located in the proximity to large blood vessels or major bile ducts, for whom standard thermal ablation techniques were prohibited [44].

1.1. IRE ablation for liver tumors. In [3, 12, Cannon et al. have shown that IRE is a safe treatment for hepatic tumors in proximity to vital structures and no liver damages have been reported in any of the patients treated.

Recently, a single-center retrospective study of the University Hospital J. Verdier at Bondy (France), on 58 patients with cirrhosis who underwent IRE ablation for 75 hepatocellular carcinoma (HCC) tumors has confirmed that IRE offers safe complete ablation of HCC tumors in patients with contraindications to other commonly used ablative techniques [41]. Therefore there is an increasing interest of interventional radiology for IRE as an alternative to standard non surgical ablation techniques.

The IRE procedure for liver tumors is standardly performed under general anesthesia as described in [16, 22]. According to the current clinical recommendations, the interventional radiologist has to place percutaneously the needles in or around the tumor in a parallel configuration and at the same depth [12]. Then the electric pulses are synchronized with electrocardiogram (ECG) and delivered thanks to a pulse generator $\mathrm{2}^{2}$. To assess the treatment success, an MRI is performed a few

\footnotetext{
${ }^{1}$ There is still a huge uncertainty on the EF magnitude threshold and on the number of micropulses needed to generate cell apoptosis, but it is commonly accepted that EF of magnitude above $500 \mathrm{~V} / \mathrm{cm}$ for several tens of $100 \mu \mathrm{s}$ pulses leads to cell death.

${ }^{2}$ There is currently only one device for clinical IRE: Nanoknife ${ }^{\circledR}$ developed by Angiodynamics, Latham, NY.
} 
days after the treatment ( 2 to 3 days), but the interpretation of the posttreatment images is still controversial [39].

1.2. Numerical treatment planning for IRE. One of the main drawbacks of IRE compared with radiofrequency $(\mathrm{RF})$ or cryo ablations lies in its technical complexity. Indeed, numerous needles (at least 3 but often 5 to 6 needles) have to be appropriately located in the vicinity of the tumor to generate the permeabilizing electric field that destroys the tumor.

To overcome this difficult but crucial task several numerical strategies have been developed to determine numerically the best needles location. One can cite the works of the groups of Miklavčič [24, 49, 48, 31, 17] and Davalos and Rubinsky [8, 6, 25, 27, 34, 35].

These groups have shown the importance of the placement of the needles to ensure the efficacy of the treatment. Their numerical simulations provide the ideal and optimal location of the needles to perform IRE to the physician. More precisely, they propose a theoretical needles configuration superimposed on the pretreatment clinical image and for which the computed electric field in the tumor reaches about $600 \mathrm{~V} / \mathrm{cm}$ [8, 27]. In these studies, the algorithms propose a strict parallel ${ }^{3}$ placement of the needles at the same depth of the tumor. These algorithms are very interesting for easily accessible tumors, namely cutaneous, sub-cutaneous, prostate or bone tumors.

However the percutaneous placement of needles to treat deep-seated tumors -as liver tumorsfaces the issue of anatomic constraints. For liver tumors located near vital structures, the numerical treatment planning strategies often propose unfeasible solution and they are thus somehow useless to the interventional radiologists. As a result, even though IRE ablation presents a lot of advantages to treat liver tumors located near vital blood vessel, it is only performed by a small community of interventional radiologists who developed an expertise beyond the paradigm that needles have to be positioned in an exactly parallel configuration and at the same depth.

Roughly speaking, the interventional radiologists who perform IRE ablation of liver tumors place the needles as best as they can, and then the pulse delivery is performed. Several clinical studies have shown the clinical relevance of this approach [12, 16, 41, 42]. However, the uncertainties of the needle positionning generate uncertainties on the electric field distribution, which may affect dramatically the treatment efficacy.

In order for IRE ablation to become a routine ablation techniques for small liver tumors near vital structures, it is therefore necessary to provide an evaluation of the treatment as it is effectively performed. Since IRE is tightly linked to the electric field distribution, it is therefore natural to compute the EF distribution in the real clinical configuration, in addition to the numerical treatment planning.

1.3. Goal of the paper. In this paper, we propose a numerical workflow for clinical IRE ablation of deep-seated tumors, to compute numerically the distribution of the electric field as generated during the procedure. We show that thanks to a combination of clinical data and numerical models of tissue electroporation it is possible to determine the electric field distribution as delivered during

\footnotetext{
${ }^{3}$ Electroporation-based therapies have been initially used to treat cutaneous or sub-cutaneous tumors, which are easily accessible. For these tumors, the placement of parallel needles, which simplifies the determination of the electric field, is quite easy.
} 
the treatment. Our numerical strategy modifies substantially the paradigm of numerics for IRE treatment. Instead of proposing an unfeasible numerical needle location, we compute the electric field in the clinical configuration, taking the real needles location.

We illustrate the relevance of our approach on a case report of a patient with hepatocellular carcinoma (HCC) who underwent IRE ablation at the University Hospital J. Verdier at Bondy, France. Interestingly, our numerical workflow makes it possible to register the numerical EF distribution on the clinical image, providing to the physician a numerical tool to assess the treatment efficacy.

\section{Material And Methods}

2.1. Clinical workflow and data collection. The clinical workflow as routinely performed at the University Hospital J. Verdier at Bondy is schematized at the top of Figure 1. The procedure has been detailed and validated by Sutter et al. [41, 42. It is somehow the minimal clinical procedure, which can be performed in most of interventional radiology operative room equipped with new generation of angiographic suit including 3D cone beam CT acquisition capacity [42]. The clinical workflow is split into 3 steps.

CT-scan (Day -30)

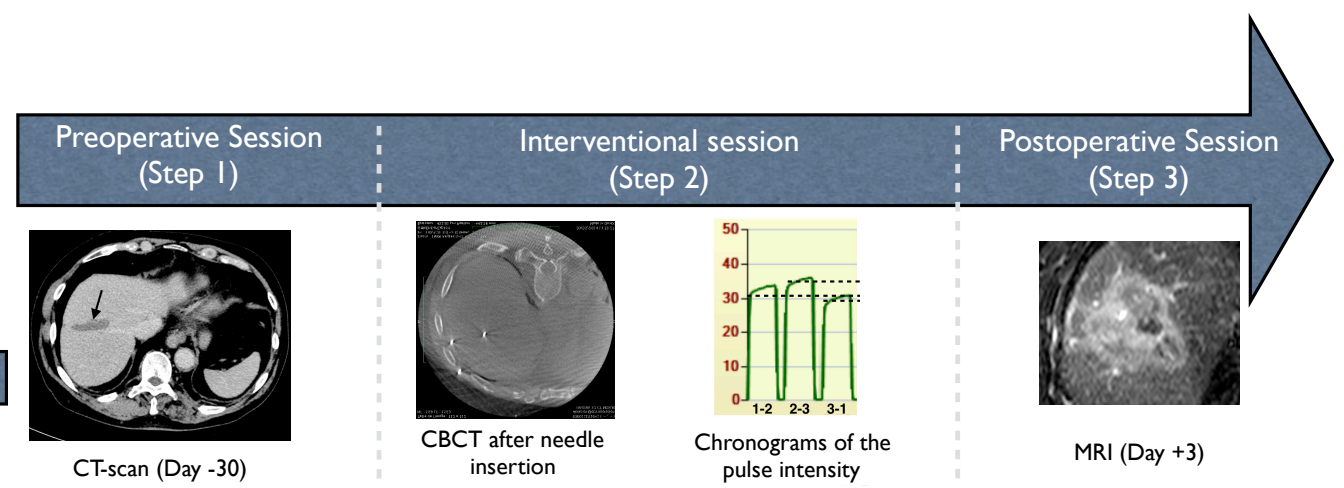

Figure 1. Typical clinical workflow [42] (top) as performed at the University Hospital J. Verdier at Bondy, France.

Clin. Step 1: The diagnostic stage provides a preoperative CT scan or MRI where the hepatic capsule, the tumor and the main liver structures are determined several days before the treatment.

Clin. Step 2: The day of the procedure, IRE ablation is performed under general anesthesia, as described by Martin et al. [36]. As previously described, the needles are percutaneously inserted around the tumor by the interventional radiologist with free-hand technic under combination of real-time ultrasound (US) and 3D Virtual Target Fluoroscopic Display such that the electric field covers the target region. The needle positioning is performed as parallel as possible. A cone beam CT (CBCT) is performed after the percutaneous insertion under combined ultrasound and fluoroscopic guidance of 2 to 6 monopolar needles around the target tumor. Then the pulse parameters are set in the pulse generator (number and 
duration of pulses, nominal electric field between the pair of active needlest...) according to the manufacturer recommendations (Nanoknife ${ }^{\circledR}$, Angiodynamics, Latham, NY). The electric pulses are then synchronized to the electrocardiogram and delivered during the refractory phase of the cardiac cycle between alternating pairs of electrodes. The pulse parameters and the chronograms of the electric voltages and intensities are recorded by the device.

Clin. Step 3: Three days after the procedure, an MRI is performed to assess the treatment efficacy.

2.2. Case report. The above clinical workflow is routinely 5 performed for HCC lesions at the University Hospital J. Verdier at Bondy, and a retrospective study confirmed its efficiency [41]. In this paper we report the case of a patient for whom a HCC tumor has been diagnosed and previously treated by radiofrequency ablation. It has left a significant scar tissue, which is the hypodense region clearly visible on the CT of Figure 2(a). One year later, blood markers indicate a suspicion of relapse, which the radiologist locates at the edge of the scar tissue (see arrow in Figure 2(a) . The IRE session is performed with 3 needles numbered from 1 to 3 , with an exposure (active tip length) of $2.5 \mathrm{~cm}$ and a spacing of about $2 \mathrm{~cm}$ following the clinical procedure described in the previous section and detailed in [42].

The protocol is 200 pulses of $70 \mu$ s per pair of electrodes (1-2, 2-3 and 3-1). During the procedure, in order to widen the treatment area, the needles are pulled backwards on $1.5 \mathrm{~cm}$. Then a second pullback of $1.5 \mathrm{~cm}$ is performed for the needles 2 and 3 . Hence, the procedure is divided into three sequences: a first train of 80 pulses per pair (including 10 test pulses), then the first pullback and a second train of 60 pulses, then the second pullback and the third train of 60 pulses per pair.

\subsection{Image Processing.}

2.3.1. ROIs Extraction. The extraction of the regions of interest (liver, tumor and main visible structures such as vessels, hepatic capsule, scars from previous treatments ...) from the preoperative imaging is performed thanks to the semi automatic segmentation tool ITK-SNAP [46]. The ROIs are then converted into masks on the computational Cartesian grid.

2.3.2. Needles positioning. The needle geometry and positions are the most sensitive data for the electric field computation. The geometry is reconstructed exactly from the manufacturer (Angiodynamics) specifications. Each needle position is recovered from the CBCT (see Step 2 of the Figure 1).

\footnotetext{
${ }^{4}$ In the Nanoknife ${ }^{\circledR}$, the distance between the needles and the nominal electric field -that is the voltage divided by the electrod distance- have to be set and the the device adjust the voltages.

${ }^{5} \mathrm{IRE}$ ablation is chosen at the University Hospital J. Verdier at Bondy for tumor lesions for which standard non surgical ablation techniques are prohibited.
} 


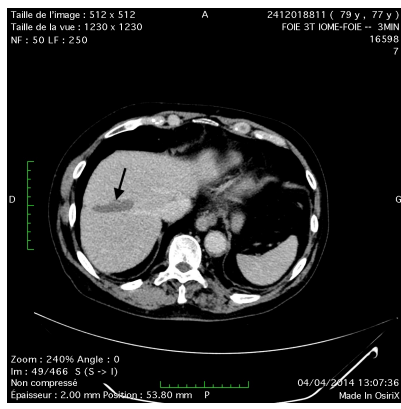

(a)

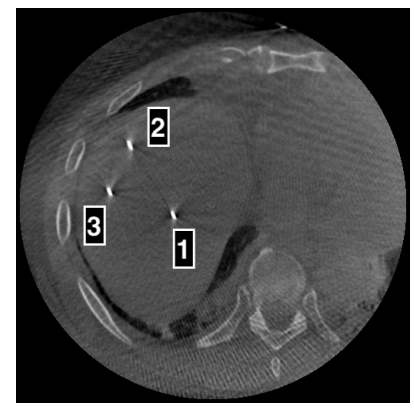

(b)

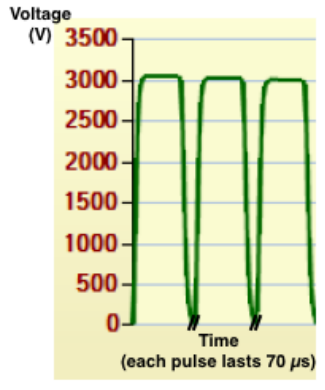

(c)

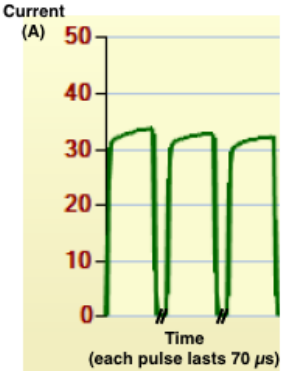

(d)

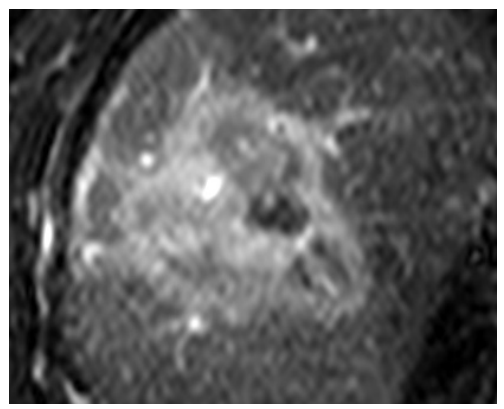

(e)

Figure 2. Clinical data of the patient case. (a): CT scan at D-45 with a scar tissue hypodense area- and a suspicion of relapse (arrow) inside the liver. (b): CBCT after needle insertion. The 3 needles are numbered from 1 to 3. (c)-(d): Voltage (c) and current (d) chronograms recorded by the Nanoknife ${ }^{\circledR}$ for one $70 \mu$ s-long pulse. For each pair needle, 60 to 80 pulses are delivered during the refractory phase of the ECG. (e): Zoom of the treated zone on the $\mathrm{T} 2$ weighted $\mathrm{MR}$ image at $\mathrm{D}+3$. One can see 3 dark areas within the high signal intensity region. They correspond to the location of the needles. Due to the high electric field, the tissue is burnt in the close vicinity of the needles. The high signal intensity zone corresponds to non burnt edematized area affected by electric field.

2.3.3. Image Registration. The CBCT with needle -see Figure 2(b) provides the geometrical framework of the pulse delivery. It is therefore the computational reference framework. The ROIs determined on the preoperative imaging are then registered on the CBCT with needles thanks to the non-rigid registration algorithm EVolution of De Senneville et al. [7, which is dedicated to multi-modal images registration.

Similarly, the ROI of the treatment area observed on the postoperative MRI are registered on the CBCT, which is thus the unique reference framework.

2.4. Numerical electroporation model. Similarly to the current numerical treatment planning computations, we chose to use the standard non linear static model which has been already extensively studied [14, 28, 32 ] 
2.4.1. Nonlinear static model. The electrostatic field $\mathbf{E}$ is derived from a potential $U$

$$
\mathbf{E}=-\nabla U
$$

The governing equation of $U$, with the medium conductivity $\sigma$, is given by the non linear electrostatic equation

$$
-\nabla \cdot(\sigma(\|\nabla U\|) \nabla U)=0
$$

where the conductivity depends nonlinearly on the magnitude of the local electric field:

$$
\sigma(|\nabla U|)=\sigma_{0}\left(1 .+a_{e p} \frac{1+\tanh \left(7.5\left(\frac{\|\nabla U\|}{E_{t h}}-1\right)\right)}{2}\right),
$$

where $\sigma_{0}$ is the conductivity the non porated tissue, $a_{e p}$ is the coefficient describing the increase of conductivity in the porated tissue, $E_{t h}$ is the threshold magnitude of the EF to generate electroporation. The threshold $E_{t h}$ is set to $300 \mathrm{~V} . \mathrm{cm}^{-1}$ [14, 28, 32].

2.4.2. Boundary conditions. Homogeneous Dirichlet and Neumann conditions are imposed on the active and inactive parts of the needles, respectively. For liver tumors located close to the lung, a homogeneous Neumann condition is imposed on the hepatic capsule. On the computational domain boundary, adjacent to the liver, a Fourier-Robin condition of parameter $\alpha=0.01$ is imposed, as a rough approximate condition of a far field boundary condition. In order to choose $\alpha$, we perform a simulation in a larger domain and we verified that the error between the two solutions was smaller than $5 \%$.

2.4.3. IRENA: a $C++$ finite difference method based software for IRE numerical assessment. Model (2) has been implemented in the $\mathrm{C}++$ software IRENA [11] developed by O. Gallinato and C. Poignard within the Inria team Monc. The code is based on the finite difference method on Cartesian grid [9]. The needles and the liver boundary are determined as the zero of a levelset function [40]. Close to the boundaries, the first and second order derivatives are computed with the standard Ghost Fluid Method [10]. The equivalent conductivities at half points are computed thanks to harmonic mean (additivity law of resistivity). In order to maintain a reasonable computation time, the refinement is low enough $\left(1 \mathrm{~mm}^{3}\right.$ grid voxels). As the mesh does not pick up the very fine needles, the Dirichlet conditions imposed on the active parts of the electrodes and the Neumann conditions imposed on the insulated parts are handled thanks to a specific adaptation of the Ghost Fluid Method. In particular, the Dirichlet conditions are changed into Robin condition thanks to a first order development of the flux. This results in a numerical scheme, which is computationally efficient and accurate enough.

2.4.4. Patient-dependent conductivities estimate obtained from the intensity chronograms. During the clinical procedure, 10 test-pulses $(70 \mu \mathrm{s}, 3000 \mathrm{~V})$ are delivered by alternating pairs of needles at the beginning of the procedure. The Nanoknife ${ }^{\circledR}$ records the chronograms of the applied voltage 
and of the intensity for all pulses. The current which flows through the needle $i$, when the electrodes $(i, j)$ are active is defined by

$$
I_{i j}=\int_{\mathcal{E}_{i}} \sigma \partial_{n} U_{i j} d x, \quad \text { for }(i j)=(1,2),(2,3),(3,1) .
$$

The detailed procedure to obtain accurately the numerical intensity is given in [11]. It is therefore possible to fit the numerical intensities on the recorded intensities to obtain the parameters $\sigma_{0}$ and $a_{e p}$ of model (2) for each region of interest (liver, tumor, scar...). We use here a simple trial and error method.

\section{Results}

3.1. Generic numerical workflow. Our numerical workflow (see Figure 3 mimics the standard clinical workflow of the subsection 2.1. It consists into 3 main numerical steps.

Num. Step 1: Segmentation of the Regions of Interest (ROIs) from the preoperative image (CT-scan or MRI) thanks to a semi-automatic segmentation before the day of the procedure.

Num. Step 2-a: Needles segmentation from the CBCT of the day of the procedure, and ROIs registration on the CBCT to get the patient-dependent geometrical framework.

Num. Step 2-b: Calibration of the electroporation model parameters from the test pulse chronograms (pre-simulations), and simulation/prediction of the treated region, thanks to the IRENA software

Num. Step 3: Registration of the numerical simulation on the early postoperative MRI.

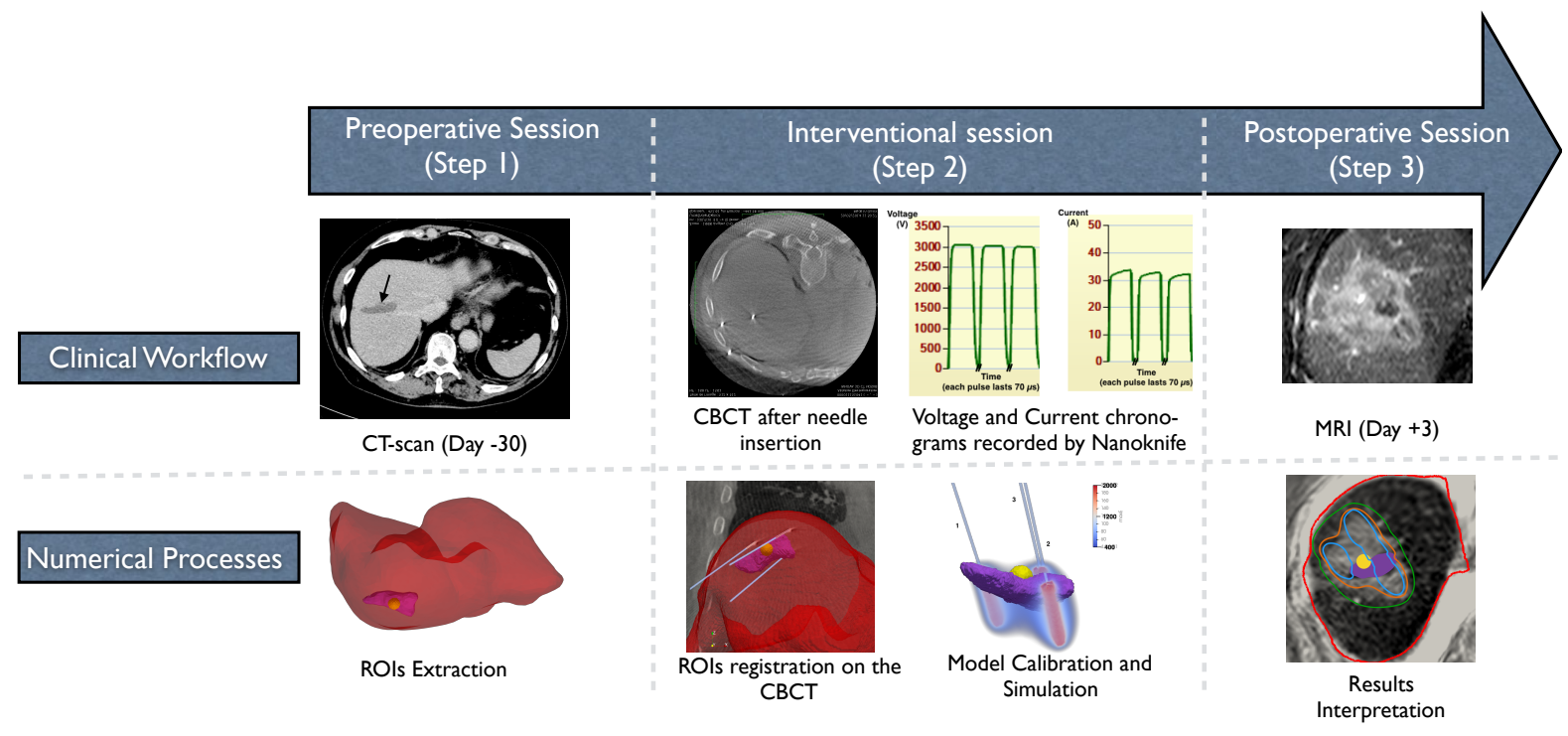

Figure 3. Our numerical workflow (bottom), which mimics the clinical workflow of Sutter et al. [42] (top). 
This numerical workflow makes it possible to determine the numerical distribution of the electric field as performed during the IRE procedure. In a near future, it could thus provide the numerical procedure-dependent electric field distribution and thus the region of the IRE ablation.

3.2. Patient-specific study. We illustrate the feasibility and the relevance of our numerical workflow on the case report presented in subsection 2.2 .

3.2.1. Segmentation of the ROIs and the needles reconstruction. In the patient study, the main constituents visible on the preoperative CT-Scan of Figure 2(a) are the hepatic capsule and the scar tissue due to a previous RF ablation. The tumor is not visible but the operator has delimited a sphere where the relapse is suspected. The segmentation of the hepatic capsule and the scar tissue have been performed with the segmentation tool ITK-SNAP [46] and given by Figure 4(a). The position of the needles are extracted from the CBCT of Figure 2(b) as shown in Figure 4(b). The red part of the needles stand for the active part where the voltage is applied, the blue parts are considered as electrically insulated.

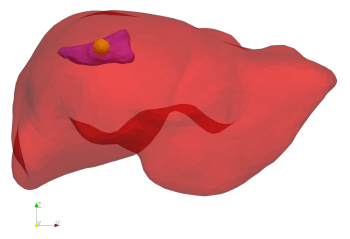

(a)

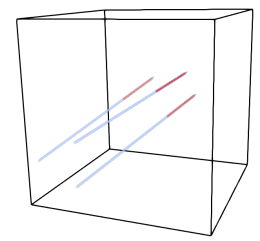

(b)

Figure 4. Geometrical framework. (a): Segmentation of the ROIs from the preoperative CT-Scan of Figure 2(a) (b): Needles reconstruction from the CBCT of Figure 2(b)

3.2.2. Registration of the pretreatment ROIs on the CBCT. In order to obtain the geometrical framework of the procedure, we performed the registration of the preoperative image on the CBCT using the EVolution algorithm [7]. This non rigid registration algorithm has been validated on clinical data and the clinical relevance of the registration has been verified by radiologists of the University Hospital J. Verdier. The deformation map of the registration is given in Figure 5(a). while a registered image is given in Figure 5(b), The EVolution algorithm provides the ROIs and the needles in the same computational domain as shown by Figure $5(\mathrm{c})$.

3.2.3. Model calibration. The strategy of the model calibration lies in the fact that the model parameters impact the value of the intensities given by equation (3). Indeed, by definition, the electric potential $U$ given by equations (2) depends on the parameters. Since the recording intensities are the only electrical data of tissue, our calibration strategy consists in fitting the numerical intensity on the recorded intensity. Using the preliminary test-pulses, we perform the calibration by a trial error method -starting from conductivity values available in the literature and changing them handly step by step to obtain the fitting-, to fit the numerical intensities with the intensities recorded by Nanoknife ${ }^{\circledR}$. The tolerance criterium of the fitting procedure is set such that during the first pulse of each pair combination, the relative error between the maximal value of the recorded 


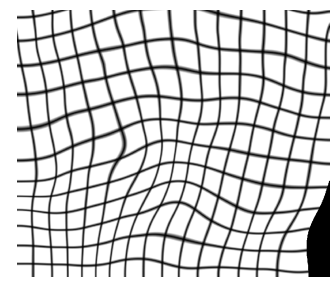

(a)

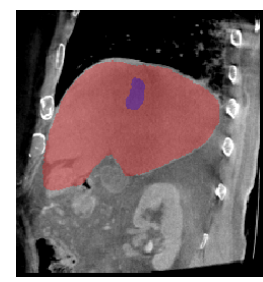

(b)

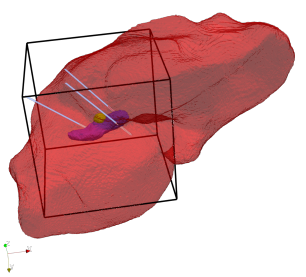

(c)

Figure 5. Non rigid registration of ROIs on the CBCT with needles performed by the EVolution algorithm [7. (a): Deformation field to register the CT-Scan on the CBCT with needles. The estimated transformation is used to deform a rectilinear regular grid. (b): ROIs of the preoperative CT-scan registered on the CBCT with needles (sagittal plane).

(c): Extraction of the 3D geometrical framework. The box is the numerical simulation box.

intensity and the simulated intensity is less than $10 \%$. It is worse noting that the calibration is performed once, with the initial needle location, and not modified afterwards.

More precisely, during the procedure the Nanoknife ${ }^{\circledR}$ records the voltage and the current for each pulse. We use these data to impose the voltage between each needle pair as given by the device, and the recorded currents are used to obtain the conductivities.

For each region of interest (liver tissue and scar tissue), we obtain the non porated tissue conductivity $\sigma_{0}$ and the coefficient $a_{e p}$. Since the tumor is not visible on the imaging, we assume that it has the same electrical property as the tissue. The scar tissue is mainly made of fibrosis, we consider that its conductivity is higher than the liver conductivity and that it is not influenced by the electric field. The results are given by Table 1 .

Table 1. Conductivity $\sigma_{0}$ and parameter $a_{e p}$ of the liver and of the scar.

\begin{tabular}{c|c|c} 
& $\sigma_{0}(\mathrm{mS} / \mathrm{cm})$ & $a_{e p}$ \\
\hline liver & 1.2 & 3. \\
scar & 2. & 0.
\end{tabular}

With these parameters, the numerical intensities are of the same order as the recorded intensities (see Figure 6).

Since the static model has been used, for each needle pair the mean intensity of the first pulse of each pair combination is fit, as shown in Figure 6. This calibration is thus a first approximation of the real conductivity of the tissues.

3.2.4. Numerical assessment of the procedure. Since the geometry and the conductivity parameters of the patient have been merely obtained, we can run a direct simulation of the IRE procedure to assess the electric field distribution in this patient-specific configuration. As Model (2) is a static model, only one pulse per pair and per sequence is simulated. For each sequence the needles pairs (1-2), (2-3) and (3-1) are alternately active. During the clinical procedure, the physician has performed 2 pullbacks of $1.5 \mathrm{~cm}$ in the coaxial direction of needles to enlarge the treated region, we 


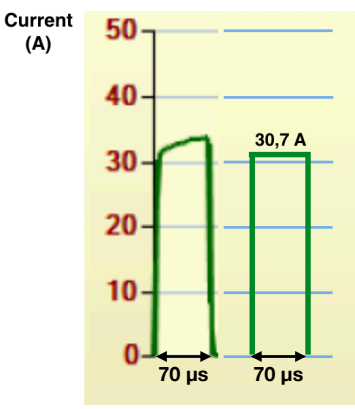

(a) needles pair 1-2

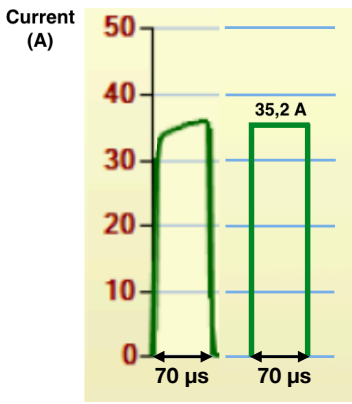

(b) needles pair 2-3

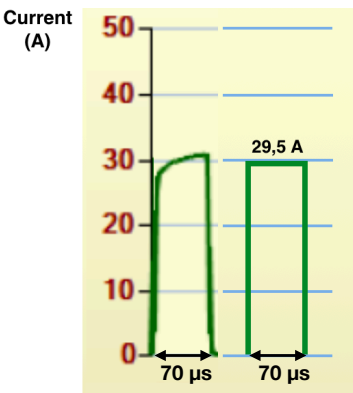

(c) needles pair 3-1

Figure 6. Numerical intensities (right) compared with the intensities recorded during the procedure (left) for 1 pulse of $70 \mu \mathrm{s}$ for each needles pair (from (a) to (c), the pairs are 1-2, 2-3 and 3-1). The applied voltage for each simulation is set to $3000 \mathrm{~V}$, which combination are given by the Nanoknife ${ }^{\circledR}$ recording.

therefore performed $3 \times 3$ simulations, which correspond to the initial geometrical configuration plus the pullbacks. Figure 7 shows the 3D distribution of the simulated electric fields at each step of the procedure.
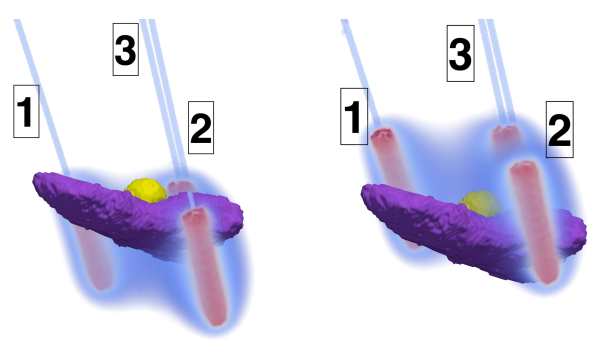

(a)

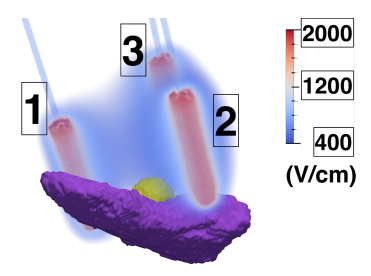

(b)

Figure 7. 3D simulation of the electric field magnitude during the whole procedure and assessment of the treated region on the CBCT. (a): Electric field magnitude (in V.cm ${ }^{-1}$ ) at each step of the procedure. Left: first pulse train delivery, Center: after the first pull-back, Right: after the second pull-back. (b): Median cross section representing the isolines of the electric field magnitude at the end of the procedure on the CBCT. The isoline $300 \mathrm{~V} \cdot \mathrm{cm}^{-1}$ is green, the isoline $500 \mathrm{~V} . \mathrm{cm}^{-1}$ is orange and the isoline $650 \mathrm{~V} . \mathrm{cm}^{-1}$ is blue. The yellow ball corresponds to the tumor location, the scar tissue is in violet.

From a medical view point, it is interesting to register the isolines of the electric field on the CBCT so that the interventional radiologist can assess the treatment efficacy. The isolines $650 \mathrm{~V} . \mathrm{cm}^{-1}$ and $300 \mathrm{~V} . \mathrm{cm}^{-1}$ are the theoretical threshold EF magnitudes needed for irreversible reversible electroporation, however since the total number of pulses at the end of the procedure is high (200 pulses are delivered), the irreversible magnitude might be lower. For instance Campelo et al. found that a electric field magnitude of $500 \mathrm{~V} \cdot \mathrm{cm}^{-1}$ could be sufficient for clinical IRE of prostate tumors [2]. Figure 7(b)] shows the isolines 650, 500 and $300 \mathrm{~V} . \mathrm{cm}^{-1}$ registered on the CBCT. 


\section{Discussion}

4.1. Choice of the electroporation model and the numerical method. For the sake of simplicity, we choose the standard non linear electrostatic model of tissue electroporation, which has already been widely studied by several groups [14, 28, 1]. Even though it is a static description, this simple model is quite well-established to provide a first estimation of the electric field distribution at the end of the pulse (in the static regime). The choice of this simple model is justified by the fact that the electrical dynamical modeling of tissue electroporation is still unclear and the current dynamic models, both cell-based and tissue-based [20, 18, 45], are still neither physically nor mathematically completely justified. Moreover the numerous parameters involved in these models still prevent their calibration and their identifiability on clinical data.

Remark 1 (On the static model of electroporation). We would like to make clear a misunderstanding which appeared in some previous studies dealing with numerical simulations of EP models. In some papers (see [28, 26, 2]) the non linear model (2) is referred to as dynamic. We emphasize that the model is static since time is not involved in the equations. Due to the nonlinearity, the numerical solution is obtained iteratively, but the iterations have nothing to do with any time evolution.

In order to benefit from the voxel structure of the clinical imaging, and the specificity of the IRE procedure (very thin needles compared to the liver, recovery of the needles location, regions of interest...) we chose to develop the $\mathrm{C}++$ code IRENA based on finite difference method. Using the voxel structure of the image and determining the regions of interest by level-sets implies that

no remeshing is necessary once the registration of the needles and the ROIs is performed, unlike the standard use of softwares based on finite element method such as COMSOL Multiphysics [5] or FreeFem $++[13]$. However the use of such softwares or others would not modify the numerical workflow.

4.2. Numerical workflow: a tool for an accurate EF distribution. The relevance of our numerical workflow lies mainly in the consideration of the procedure specificities. In particular, the needles location and the liver conductivities are essential. It is worth noting that the segmentation of the liver capsule can be skipped if the tumor is far enough from it. Conversely supplementary influent structures as arterial or biliar trees can be added if they are visible on the pretreatment imaging. Their segmentation and registration do not differ from what has been described in this study.

The reconstruction of the specific geometry of the procedure inevitably produces errors. For instance, the segmentation strongly depends on the imaging resolution, and on the operator. The numerical errors related to the registration, the discretization and the resolution of the mathematical problem, are numerically controlled and quantifiable. The modeling assumptions for the registration are not discussed here. For more details, we refer the reader to [7].

The electric current graphs are the only clinical data providing valuable information on the electrical property of the tissues. They are therefore essential for the calibration stage. While the values found in literature are rather variable and in wide ranges of values, the conductivity 
parameters of the test-case are directly calibrated from the current graphs. Hence, the relevance of our clinical workflow also relies on this patient-dependent calibration thanks to the use of the recorded intensities during the procedure.

4.3. Issue of treatment assessment. The assessment of the treatment efficacy is a hard task. Apoptosis is a complex biological proces\$ ${ }^{6}$ that occurs several hours after IRE procedure [37]. The manufacturer of the Nanoknife ${ }^{\circledR}$ (Angiodynamics, Latham, NY) states that a significant increase in the electrical intensity (of the order of $10 \mathrm{~A}$ ) ensures an irreversible electroporation of the tissue. This choice of intensity increase is somehow arbitrary. Most of the publications consider that the electric field threshold for IRE lies between 500 and $700 \mathrm{~V} . \mathrm{cm}^{-1}$. More precisely, experimental observations say the EF magnitude above $600 \mathrm{~V} \cdot \mathrm{cm}^{-1}$ is necessary [23, 6, 15], but Campelo et al. found a lower threshold value (around $500 \mathrm{~V} . \mathrm{cm}^{-1}$ ) for a clinical IRE procedure in the prostate [2].

This question can be explored by comparing the results of simulations with the post procedure imaging. Indeed, the early postoperative MRI shows an impact of the treatment 3 days after the procedure, as shown in Figure 2(e) on the T2 weighted image. However the clinical interpretation of this imaging is still controversial. In particular, it is not clear whether the hyperdense region observed on Figure 2(e) is a reversibly or irreversibly permeabilized region.

Interestingly, our numerical simulations can provide new keys for the interpretation of the different modalities. Since the simulations have been performed in the frame of the CBCT, we registered the post-treatment MRI in this frame too, thanks to the EVolution algorithm. Then, simulations are superimposed on the registered post-treatment MRI. The results are given in Figure 8 ,

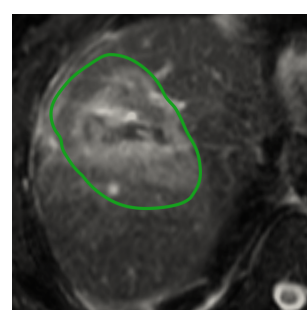

(a)

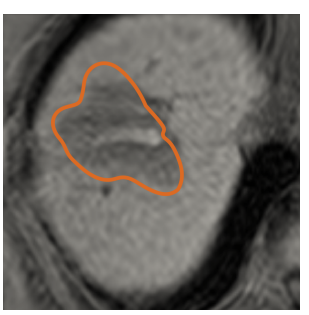

(b)

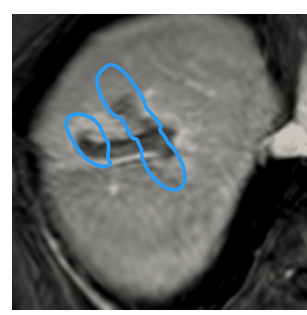

(c)

Figure 8. Cross section of the isolines 300,500 and $650 \mathrm{~V} . \mathrm{cm}^{-1}$ registered respectively on $\mathrm{T} 2$ (a), unenhanced (b) and enhanced at delayed phase of intravenous injection of gadolinium (c) of $\mathrm{T} 1$ weighted images.

As shown in Figure 8, each modality enhances a specific feature of the treatment, which can be correlated to the simulation. More precisely, the T2 weighted image exhibits the largest zone affected by the treatment. According to our simulation (see Figure 8(a)], this zone is included in the zone delimited by the isoline $300 \mathrm{~V} \cdot \mathrm{cm}^{-1}$. On the $\mathrm{T} 1$ weighted image (see Figure 8(b) (Center)), the limit of the hypodense area seems correlated to the isoline $500 \mathrm{~V} . \mathrm{cm}^{-1}$. It probably corresponds to the largest area where the cells are killed by the electric field. Eventually, on the

\footnotetext{
${ }^{6}$ It probably results from the loss of cellular material due to a large and persistent permeabilization of cell membranes, following their oxidation during poration [30, 43, 20, 33].
} 
delayed phase of gadolinium-enhanced T1 weighted image (see Figure 8(c)], the hypodense region exhibits the scar and 3 spots where the needles were located. This is probably the region where the tissue has been burnt due to the pulse delivery. It is worth noting that this region is in the close vicinity of the electrodes. Of course this possible new lecture of MRI modalities has to be confirmed on a large panel of clinical cases.

\section{Conclusion}

This paper shows on a specific clinical case that a combination of the clinical data with numerical approaches make it possible to predict the electric field distribution from clinical data and to correlate the electroporated regions with the post treatment MRI. Therefore it somehow paves the way of a real-time patient-dependent determination of the IRE ablation region, thanks to a combination of numerical models, image registration techniques and clinical data. Our contributions consist of the three next points:

(1) A numerical workflow based on the clinical workflow as performed at the University Hospital J. Verdier at Bondy. It consists of image registration, electroporation (EP) model calibration, and numerical simulation.

(2) A model calibration strategy from clinical data.

(3) A proof of concept on a specific clinical case of a patient with HCC who underwent IRE ablation.

The proof of concept based on a patient-specific case gives an interesting basis for confronting models to clinical cases in order to improve understanding and modeling, in a retrospective approach.

The accurate numerical reconstruction of the clinical framework is obtained thanks to advanced image segmentation and registration of clinical data provided in standard IRE procedures. The conductivity calibration thanks to the recorded current during the procedure is a promising direction to be further investigated.

The numerical workflow based on clinical data provides realistic simulations of the EF distribution, thanks to a standard electroporation tissue model. The numerical results are corroborated afterwards on early postoperative imaging.

Thanks to the registration of the numerical simulations and the post-treatment MRI in the same frame, we propose a new lecture of the post treatment MRI. More precisely, the T2 weighted image seems to give an estimation of the electric field distribution and thus of the maximal area affected by the electric field, the T1 weighted image could give information on the irreversibly permeabilised area. The enhanced at delayed phase of intravenous injection of gadolinium of T1 weighted image seems to exhibit the necrosis generated by the treatment in the close vicinity of the needle. This paper is somehow a proof of concept of a possible computational strategy for tumor IRE ablation. Of course the study needs further investigations on a large cohort of patients with HCC treated by IRE in order to evaluate the advantages and the accuracy of our method, and to confirm our results. 
Statement of ethics board approval. This retrospective study is in accordance with ethical principals of the Declaration of Helsinki and has been approved by the local committee on human research of the University Hospital J. Verdier.

\section{ACKNOWLEDGEMENT}

The authors thank very warmly the referees for their helpful comments and suggestions, that help to improve the manusript. This study has been carried out within the frame of the LABEX TRAIL, ANR-10-LABX-0057 with financial support from the French State, managed by the French National Research Agency (ANR) in the frame of the "Investments for the future" Programme IdEx (ANR10-IDEX-03-02). The authors are supported by the INCA Aviesan Plan Cancer projects DYNAMO (ref. PC201615) and NUMEP (ref. PC201615).

\section{REFERENCES}

[1] M. Breton, F. Buret, L. Krähenbühl, M. Leguèbe, L. M. Mir, R. Perrussel, C. Poignard, R. Scorretti, and D. Voyer. Non-Linear Steady-State Electrical Current Modeling for the Electropermeabilization of Biological Tissue. IEEE Transactions on Magnetics, 51(3):1-4, Mar. 2015.

[2] S. Campelo, M. Valerio, H. U. Ahmed, Y. Hu, S. L. Arena, R. E. Neal, M. Emberton, and C. B. Arena. An evaluation of irreversible electroporation thresholds in human prostate cancer and potential correlations to physiological measurements. APL Bioengineering, 1(1):016101, Dec. 2017.

[3] R. Cannon, S. Ellis, D. Hayes, G. Narayanan, and R. C. Martin. Safety and early efficacy of irreversible electroporation for hepatic tumors in proximity to vital structures: IRE for Hepatic Tumors. Journal of Surgical Oncology, 107(5):544-549, Apr. 2013.

[4] K. P. Charpentier, F. Wolf, L. Noble, B. Winn, M. Resnick, and D. E. Dupuy. Irreversible electroporation of the liver and liver hilum in swine. HPB, 13(3):168-173, Mar. 2011.

[5] COMSOL AB, Stockholm, Sweden. Comsol multiphysics ${ }^{\circledR}$.

[6] R. V. Davalos, L. M. Mir, and B. Rubinsky. Tissue Ablation with Irreversible Electroporation. Annals of Biomedical Engineering, 33(2):223-231, Feb. 2005.

[7] B. Denis de Senneville, C. Zachiu, M. Ries, and C. Moonen. EVolution: an edge-based variational method for non-rigid multi-modal image registration. Physics in Medicine and Biology, 61(20):7377-7396, Oct. 2016.

[8] J. F. Edd and R. V. Davalos. Mathematical modeling of irreversible electroporation for treatment planning. Technology in cancer research \& treatment, 6(4):275-286, 2007.

[9] R. Eymard, T. Gallouët, and R. Herbin. Finite volume methods. In Handbook of numerical analysis, Vol. VII, Handb. Numer. Anal., VII, pages 713-1020. North-Holland, Amsterdam, 2000.

[10] R. P. Fedkiw, T. Aslam, B. Merriman, and S. Osher. A Non-oscillatory Eulerian Approach to Interfaces in Multimaterial Flows (the Ghost Fluid Method). Journal of Computational Physics, 152(2):457-492, 1999.

[11] O. Gallinato and C. Poignard. IRENA: a Finite Volume Method based software for the numerical assessment of clinical IRE.

[12] D. A. Gervais, S. N. Goldberg, D. B. Brown, M. C. Soulen, S. F. Millward, and D. K. Rajan. Society of interventional radiology position statement on percutaneous radiofrequency ablation for the treatment of liver tumors. Journal of Vascular and Interventional Radiology, 20, 2009.

[13] F. Hecht. New development in freefem++. J. Numer. Math., 20(3-4):251-265, 2012.

[14] A. Ivorra, L. M. Mir, and B. Rubinsky. Electric field redistribution due to conductivity changes during tissue electroporation: experiments with a simple vegetal model. In World Congress on Medical Physics and Biomedical Engineering, September 7-12, 2009, Munich, Germany, pages 59-62. Springer, 2009.

[15] C. Jiang, R. V. Davalos, and J. C. Bischof. A Review of Basic to Clinical Studies of Irreversible Electroporation Therapy. IEEE Transactions on Biomedical Engineering, 62(1):4-20, Jan. 2015. 
[16] N. Jourabchi, K. Beroukhim, B. A. Tafti, S. T. Kee, and E. W. Lee. Irreversible electroporation (nanoknife) in cancer treatment. Gastrointestinal Intervention, 3(1):8 - 18, 2014.

[17] B. Kos, P. Voigt, D. Miklavčič, and M. Moche. Careful treatment planning enables safe ablation of liver tumors adjacent to major blood vessels by percutaneous irreversible electroporation (IRE). raon, 49(3):234, 2015.

[18] J. Langus, M. Kranjc, B. Kos, T. Šuštar, and D. Miklavčič. Dynamic finite-element model for efficient modelling of electric currents in electroporated tissue. Scientific Reports, 6(1), Sept. 2016.

[19] E. W. Lee, C. Chen, V. E. Prieto, S. M. Dry, C. T. Loh, and S. T. Kee. Advanced Hepatic Ablation Technique for Creating Complete Cell Death: Irreversible Electroporation. Radiology, 255(2):426-433, May 2010.

[20] M. Leguèbe, A. Silve, L. Mir, and C. Poignard. Conducting and permeable states of cell membrane submitted to high voltage pulses: Mathematical and numerical studies validated by the experiments. Journal of Theoretical Biology, 360:83-94, Nov. 2014.

[21] E. Maor, A. Ivorra, J. Leor, and B. Rubinsky. The effect of irreversible electroporation on blood vessels. Technology in cancer research \& treatment, 6(4):307-312, 2007.

[22] R. Martin, E. Schwartz, J. Adams, I. Farah, and B. Derhake. Intra - operative anesthesia management in patients undergoing surgical irreversible electroporation of the pancreas, liver, kidney, and retroperitoneal tumors. Anesthesiology and Pain Medicine, 2015.

[23] D. Miklavčič, D. Šemrov, H. Mekid, and L. M. Mir. A validated model of in vivo electric field distribution in tissues for electrochemotherapy and for DNA electrotransfer for gene therapy. Biochimica et Biophysica Acta (BBA) - General Subjects, 1523(1):73 - 83, 2000.

[24] D. Miklavčič, M. Snoj, A. Županič, B. Kos, M. Čemažar, M. Kropivnik, M. Bracko, T. Pečnik, E. Gadzijev, and G. Serša. Towards treatment planning and treatment of deep-seated solid tumors by electrochemotherapy. BioMedical Engineering OnLine, 9(1):10, 2010.

[25] R. E. Neal and R. V. Davalos. The Feasibility of Irreversible Electroporation for the Treatment of Breast Cancer and Other Heterogeneous Systems. Annals of Biomedical Engineering, 37(12):2615-2625, Dec. 2009.

[26] R. E. Neal, P. A. Garcia, H. Kavnoudias, F. Rosenfeldt, C. A. Mclean, V. Earl, J. Bergman, R. V. Davalos, and K. R. Thomson. In Vivo Irreversible Electroporation Kidney Ablation: Experimentally Correlated Numerical Models. IEEE Transactions on Biomedical Engineering, 62(2):561-569, Feb. 2015.

[27] R. E. Neal, P. A. Garcia, J. L. Robertson, and R. V. Davalos. Experimental Characterization and Numerical Modeling of Tissue Electrical Conductivity during Pulsed Electric Fields for Irreversible Electroporation Treatment Planning. IEEE Transactions on Biomedical Engineering, 59(4):1076-1085, Apr. 2012.

[28] R. E. Neal, J. L. Millar, H. Kavnoudias, P. Royce, F. Rosenfeldt, A. Pham, R. Smith, R. V. Davalos, and K. R. Thomson. In vivo characterization and numerical simulation of prostate properties for non-thermal irreversible electroporation ablation: Characterized and Simulated Prostate IRE. The Prostate, 74(5):458-468, May 2014.

[29] C. Niessen, S. Thumann, L. Beyer, B. Pregler, J. Kramer, S. Lang, A. Teufel, E. M. Jung, C. Stroszczynski, and P. Wiggermann. Percutaneous Irreversible Electroporation: Long-term survival analysis of 71 patients with inoperable malignant hepatic tumors. Scientific Reports, 7:43687, Mar. 2017.

[30] S. Orlowski and L. M. Mir. Cell electropermeabilization: a new tool for biochemical and pharmacological studies. Biochimica et Biophysica Acta (BBA)-Reviews on Biomembranes, 1154(1):51-63, 1993.

[31] D. Pavliha, M. M. Mušič, G. Serša, and D. Miklavčič. Electroporation-Based Treatment Planning for Deep-Seated Tumors Based on Automatic Liver Segmentation of MRI Images. PLoS ONE, 8(8):e69068, Aug. 2013.

[32] R. Qasrawi, L. Silve, F. Burdío, Z. Abdeen, and A. Ivorra. Anatomically Realistic Simulations of Liver Ablation by Irreversible Electroporation: Impact of Blood Vessels on Ablation Volumes and Undertreatment. Technology in Cancer Research \& Treatment, 16(6):783-792, Dec. 2017.

[33] C. Rosazza, S. Haberl Meglič, A. Zumbusch, M.-P. Rols, and D. Miklavčič. Gene electrotransfer: a mechanistic perspective. Current gene therapy, 16(2):98-129, 2016.

[34] B. Rubinsky and J. Nagel, editors. Irreversible Electroporation. Series in Biomedical Engineering. Springer Berlin Heidelberg, Berlin, Heidelberg, 2010. 
[35] B. Rubinsky, G. Onik, and P. Mikus. Irreversible electroporation: a new ablation modality-clinical implications. Technology in cancer research $\&$ treatment, 6(1):37-48, 2007.

[36] J. Rubinsky, G. Onik, P. Mikus, and B. Rubinsky. Optimal Parameters for the Destruction of Prostate Cancer Using Irreversible Electroporation. The Journal of Urology, 180(6):2668-2674, 2008.

[37] A. Saraste. Morphologic and biochemical hallmarks of apoptosis. Cardiovascular Research, 45(3):528-537, Feb. 2000.

[38] H. J. Scheffer, K. Nielsen, M. C. de Jong, A. A. van Tilborg, J. M. Vieveen, A. R. Bouwman, S. Meijer, C. van Kuijk, P. M. van den Tol, and M. R. Meijerink. Irreversible Electroporation for Nonthermal Tumor Ablation in the Clinical Setting: A Systematic Review of Safety and Efficacy. Journal of Vascular and Interventional Radiology, 25(7):997-1011, July 2014.

[39] O. Seror, C. Poignard, O. Gallinato, R. Ourabia-Belkacem, O. Sutter, and S. A. Padia. Irreversible Electroporation: Disappearance of Observable Changes at Imaging Does Not Always Imply Complete Reversibility of the Underlying Causal Tissue Changes. Radiology, 282(1):301-302, 2017.

[40] J. A. Sethian. Level set methods, volume 3 of Cambridge Monographs on Applied and Computational Mathematics. Cambridge University Press, Cambridge, 1996. Evolving interfaces in geometry, fluid mechanics, computer vision, and materials science.

[41] O. Sutter, J. Calvo, G. N'Kontchou, J.-C. Nault, R. Ourabia-Belkacem, P. Nahon, N. Ganne-Carrié, V. Bourcier, N. Zentar, F. Bouhafs, N. Sellier, A. Diallo, and O. Seror. Safety and Efficacy of Irreversible Electroporation for the Treatment of Hepatocellular Carcinoma Not Amenable to Thermal Ablation Techniques: A Retrospective Single-Center Case Series. Radiology, 284(3):877-886, Sept. 2017.

[42] O. Sutter, A. Fihri, R. Ourabia-Belkacem, N. Sellier, A. Diallo, and O. Seror. Real-time 3d virtual target fluoroscopic display for challenging hepatocellular carcinoma ablations using cone beam CT. Technology in Cancer Research \& Treatment, 17, 2018.

[43] J. Teissié, M. Golzio, and M. P. Rols. Mechanisms of cell membrane electropermeabilization: A minireview of our present (lack of ?) knowledge. Biochimica et Biophysica Acta (BBA) - General Subjects, 1724(3):270 - 280, 2005.

[44] K. R. Thomson, W. Cheung, S. J. Ellis, D. Federman, H. Kavnoudias, D. Loader-Oliver, S. Roberts, P. Evans, and C. Ball. Investigation of the safety of irreversible electroporation in humans. Journal of Vascular and Interventional Radiology, 22, 2011.

[45] D. Voyer, A. Silve, L. M. Mir, R. Scorretti, and C. Poignard. Dynamical modeling of tissue electroporation. Bioelectrochemistry, 119:98-110, Feb. 2018.

[46] P. A. Yushkevich, J. Piven, H. Cody Hazlett, R. Gimpel Smith, S. Ho, J. C. Gee, and G. Gerig. User-guided 3D active contour segmentation of anatomical structures: Significantly improved efficiency and reliability. Neuroimage, 31(3):1116-1128, 2006. www.itksnap.org.

[47] A. Zimmerman, D. Grand, and K. Charpentier. Irreversible electroporation of hepatocellular carcinoma: patient selection and perspectives. Journal of Hepatocellular Carcinoma, Volume 4:49-58, Mar. 2017.

[48] A. Županič, B. Kos, and D. Miklavčič. Treatment planning of electroporation-based medical interventions: electrochemotherapy, gene electrotransfer and irreversible electroporation. Physics in Medicine and Biology, 57(17):5425-5440, Sept. 2012.

[49] A. Županič and D. Miklavčič. Optimization and numerical modeling in irreversible electroporation treatment planning. In Irreversible electroporation, pages 203-222. Springer, 2010. 